\title{
Eindrücke aus dem Biebrza-Nationalpark in Polen
}

\author{
HiLKe STEINECKE
}

\begin{abstract}
The Biebrza national park, founded in 1993, is the largest national park of Poland. The Biebrza river valley is the most natural complex of fens and bogs in middle and western Europe. The vegetation of the river valley is very diverse. It is dominated by communities of low plants, sedge communities, moss-sedge and meadow vegetation. Some plants like Polemonium caeruleum are relicts of ice age. The Biebrza marshes are a place from where the last elks of Poland survived and spread all over the country. Some characteristic plants and animals are described.
\end{abstract}

\section{Zusammenfassung}

Der Biebrza-Nationalpark wurde 1993 gegründet und ist der größte Nationalpark Polens. Das Biebrza-Flusstal ist eine der wenigen ursprünglichen Landschaften Mittel- und Westeuropas. Die Vegetation im Flusstal ist vielfältig. Es dominiert offene Vegetation mit niedrigen Pflanzen, häufig sind Seggenrieder, Moos-Seggen-Gemeinschaften und Weideland. Manche Arten wie z. B. Polemonium caeruleum sind Relikte aus der Eiszeit. Die Biebrza-Sümpfe waren Rückzugsgebiet der in Polen fast ausgerotteten Elche. Von dort konnten sie sich wieder über das ganze Land ausbreiten.

\section{Einführung}

Der Biebrza-Nationalpark (polnisch: Biebrzánski Park Narodowy) ist mit einer Fläche von knapp $600 \mathrm{~km}^{2}$ und einer Länge von etwa $100 \mathrm{~km}$ der größte Nationalpark Polens. Er befindet sich im Nordosten Polens am Rande von polnischen, litauischen und russischen Kulturgebieten in der Tiefebene Podlaskie. Da sich vermutlich viele, die nicht der polnischen Sprache mächtig sind, mit der Aussprache des polnischen Namens schwer tun, kann als Hilfestellung erwähnt werden, dass der Biebrza-Fluss in deutscher Übersetzung Biber-Fluss heißt.

Aufgrund der Lage im östlichen Mitteleuropa wird das Flusstal durch kontinentales Klima beeinflusst und ist eines der kältesten Gebiete Polens. Die Winter sind hart und es gibt ca. 110 Tage pro Jahr mit Schneebedeckung. Der Sommer ist kurz, aber warm.

Schon nach dem ersten Weltkrieg wurden hier zwei Schutzgebiete ausgewiesen (Czervone Bagno und Grzedy), aber erst 1993 wurde der Nationalpark in seiner heutigen Form gegründet. Dominiert wird er von dem Fluss Biebrza, einem der letzen frei fließenden, stark mäandrierenden Flüsse Europas und den angrenzenden weiten, nahezu unzugänglichen Sumpf- und Moorgebieten. Bedeutendes und besonders streng geschütztes Sumpfgebiet ist der Rote Sumpf (polnisch: Czerwone Bagno).
In den Sumpfgebieten ist eine für Mitteleuropa extrem reiche Pflanzen- und Tierwelt mit vielen bedrohten Arten anzutreffen, von denen nachfolgend einige kurz vorgestellt werden sollen. Unter den Pflanzen konnten sich ReliktArten aus der Eiszeit halten. Biber, Wölfe sowie Elche sind hier bemerkenswerte Tiere. Der Nationalpark ist zudem ein bedeutendes Rückzugsgebiet für zahlreiche seltene Vogel-Arten. Ein charakteristischer Vogel ist der Kampfläufer, der auch das Logo des Nationalparks ziert. Aufgrund dieser Artenvielfalt sowie der landschaftlichen Besonderheit wurde das Gebiet 1995 auf die RAMSAR-Konventionsliste der wichtigsten schützenswerten Feuchtgebiete der Erde gesetzt.

Der Nationalpark ist von fünf kleinen Dörfern besiedelt. In diesen kann sich der Besucher ein gutes Bild von der traditionellen landwirtschaftlichen Nutzung des Gebietes machen. Knapp die Hälfte der Parkfläche findet sich in Privatbesitz. Die Bauern nutzen ihre feuchten Wiesen entlang des Flusses überwiegend als Weideland für Milchvieh. Die alljährlich wiederkehrenden Überschwemmungen werden nicht als Bedrohung aufgefasst, vielmehr hat man sich gut damit arrangiert. Da Hochwasser vermutlich regelmäßig alle Weidezäune einreißen würde, gibt es keine eingezäunten Weidegründe, so dass die Kühe frei herumlaufen dürfen. Auf Außenstehende wirkt es äußerst romantisch, 


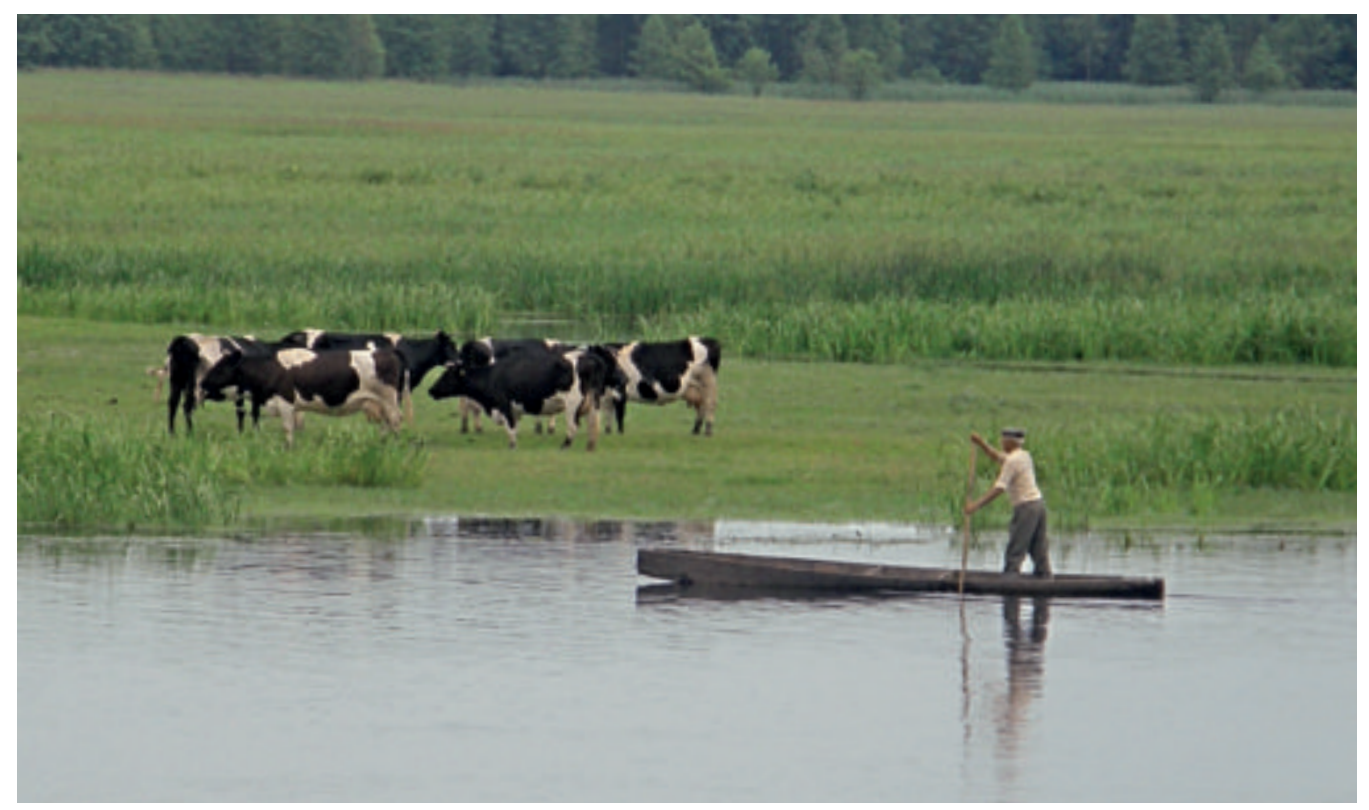

wenn Bauern auf ihren kleinen Kähnen den Fluss sowie überschwemmte Flächen überqueren müssen, wenn sie ihr Vieh zusammentreiben wollen. Schilf und Seggen werden noch als Rohstoffe genutzt, mit denen Hausdächer gedeckt werden. Als Baumaterial verwendet werden auch die einst von eiszeitlichen Gletschern aus dem Norden herantransportierten Findlinge. Die ortsansässige Bevölkerung trägt mit ihrer traditionellen Nutzung zusammen mit der Nationalparkverwaltung sowie nationalen und internationalen Umweltschutzorganisationen, darunter auch dem WWF Deutschland, zum Schutz dieser einzigartigen Naturlandschaft bei.

In den letzten Jahren wurde Infrastruktur für den Ökotourismus und die Umweltbildung geschaffen. Es gibt 14 Wanderwege mit einer Gesamtlänge von ca. $400 \mathrm{~km}$, einen rund $20 \mathrm{~km}$ langen Fahrradweg, Tierbeobachtungskanzeln, Aussichtstürme, Holzstege in Sümpfe sowie Informationstafeln zur Tier- und Pflanzenwelt. Auf dem Wasserwege sind ein- bis mehrtägige Kanutouren möglich. Direkt neben dem Roten Sumpf am Rande des Dorfes Wolnawie wurde erst vor wenigen Jahren ein Holz-Hotel (Zagroda

Abb. 1: Milchkühe am Biebrza-Fluss.
Kuwasy) im Stil eines Landgutes errichtet. Es bietet sich als Ausgangspunkt für Touren in den Nationalpark an. Hier sind auch Karten, Nationalpark-Informationen und Eintrittskarten erhältlich.

Die Kerngebiete des Nationalparks sind nur mit einem sach- und ortskundigen Führer zu betreten. Zu diesem Zweck wurden in den letzten Jahren 90 Nationalparkführer ausgebildet. Jährlich besuchen etwa 30000 Menschen den Biebrza-Nationalpark. Ein großer Anteil der Touristen stammt aus Deutschland und kommt vor allem aus ornithologischem Interesse. Daneben sind aber auch viele polnische Schulgruppen im Gebiet unterwegs.

\section{Der Biebrza-Fluss}

Der Fluss hat eine Länge von 164 km, das Flusstal mit den vielen veränderlichen Mäandern und Altwassern mitsamt der Überschwemmungsgebiete umfasst eine Fläche von gut $7000 \mathrm{~km}^{2}$. Als typischer Niederungsfluss schlängelt er sich in unzähligen Schleifen durch die Landschaft, seine Tiefe variiert von 0,5 bis mehrere Meter. Große Flächen des Flussbettes werden in jedem Frühjahr überschwemmt, so dass der Eindruck eines großen Sees entsteht. Das Flusstal ist von einer mehrere Meter dicken Torfschicht bedeckt, 

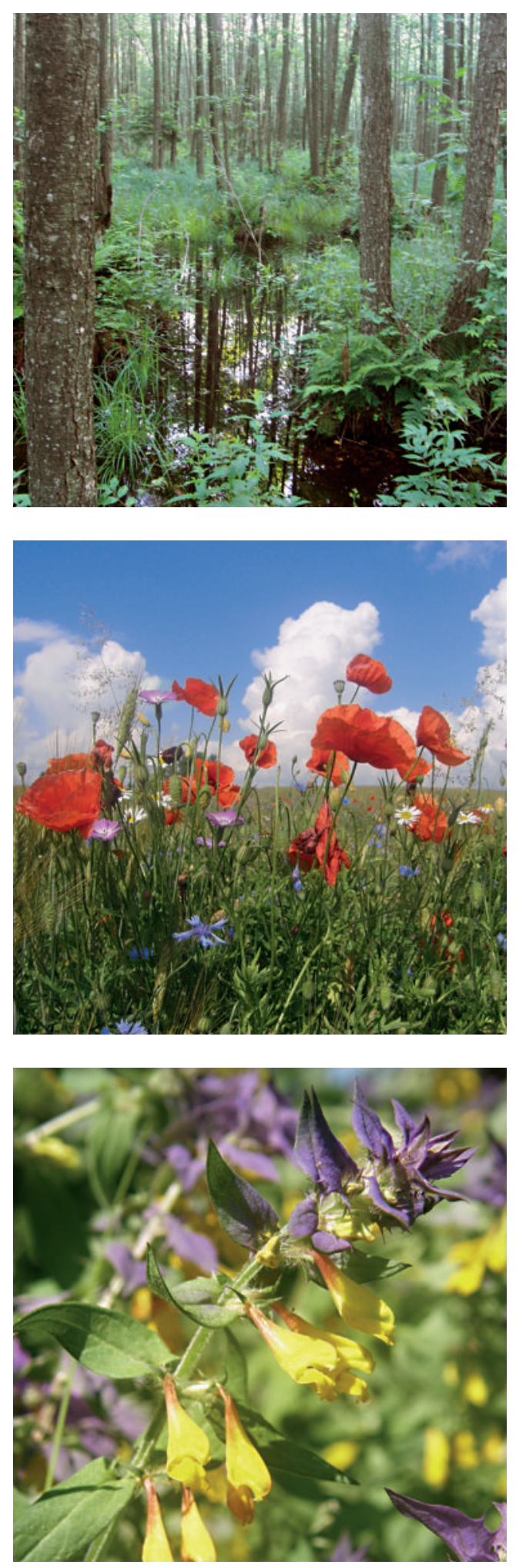

womit es das größte ursprünglichen Torfmoorgebiet in Mitteleuropa ist.

Trotz allem ist diese Flusslandschaft nicht gänzlich von menschlichen Einflüssen unberührt. Schon vor etwa 500 Jahren haben Menschen erste bedeutende Eingriffe in die Natur des Flusstals vorgenommen: einzelne Flecken im Sumpfwald wurden gerodet und in Torfwiesen zur Heugewinnung umgewandelt. $\mathrm{Zu}$ Beginn des 19. Jh. versuchten Wasserbauer, den Fluss zu zähmen und durch Anlegen von Kanälen und Begradigungen die Infrastruktur von Verkehrswegen zu verbessern; im Jahr 1824 wurde mit dem Bau des Augustowski-Kanals begonnen. Unter Einbezug verschiedener Flüsse sollte eine Wasserverbindung zwischen Polen, der Baltischen Küste und Danzig geschaffen werden, um den Warentransport zwischen diesen Regionen $\mathrm{zu}$ vereinfachen. In diesem Zusammenhang wurde auch das Flussbett vertieft. Aufgrund dieser Veränderungen sank der Grundwasserspiegel und wurde die Neubildung von Torfmoos beeinträchtigt, wodurch sich die Vegetation stellenweise in Richtung trockenheitsliebendere Pflanzengemeinschaften veränderte und sich dort, wo sich der Torf zersetzte, Mineralstoffe ansammelten.

Auch Kriege haben ihre Spuren hinterlassen, denn die undurchdringlichen Sümpfe dienten als natürliche Verteidigungslinie: Aus der Zarenzeit (19. Jh.) stammt die Festung Osowiec und die Molotow-Linie ist ein Zeitzeuge des Zweiten Weltkriegs.

\section{Pflanzenwelt}

Bereits die Pufferzone rund um den Nationalpark bietet für den Naturfreund viele Besonderheiten. Im ländlich geprägten Raum wechseln sich kleine Dörfer, Felder, Wiesen, Seen und Kiefern- bzw. Birkenwälder ab. Noch werden in dieser Region vermutlich weniger Herbizide als in Deutschland verwendet. Dementsprechend

Abb. 2 (oben): Im Roten Sumpf.

Abb. 3 (Mitte): Bunte Ackerwildkräuter: Klatschmohn, Kamille und Kornrade.

Abb. 4 (unten): Melampyrum nemorosum. 
gibt es hier noch reichlich Ackerwildkräuter wie Acker-Rittersporn (Consolida regalis), Kornblume (Centaurea cyanus) und Klatschmohn (Papaver rhoeas), die in Deutschland stellenweise sehr selten geworden sind. Bei uns sucht man z. B. die Kornrade (Agrostemma githago) auf Äckern vergeblich, gelegentlich wird sie als einjährige Sommerblume oder in Schmetterlingswiesen ausgesät. Sie wurde in der Mitte des 20. Jhs. besonders stark bekämpft, weil ihre toxischen Samen früher häufig das Getreide verunreinigten und somit auch das entsprechende Mehl und die daraus gebackenen Brote giftig waren. Im Nordosten Polens dagegen ist die Kornrade auf vielen Äckern ein alltägliches Bild. An Weg- und Feldrändern sind zudem Seifenkraut und der Halbparasit Hain-Wachtelweizen (Melampyrum nemorosum) mit seinen gelben Blüten und den violetten Hochblättern keine Seltenheit. Die von Europa bis Sibirien heimische einjährige Pflanze parasitiert auf Gräsern und entzieht ihnen Wasser und Nährstoffe. Manchmal fühlt sich der Polen-Reisende in die gute alte Zeit zurückversetzt, wenn man beobachtet, wie Bauern noch einfache Pferdekarren als Transportmittel benutzen.

Im Nationalpark nehmen die Wälder 26\% der Fläche ein, offene Lebensräume wie Wiesen, Sümpfe, Moore, Röhrichte und Seggenrieder sind mit $70 \%$ vertreten, die Wasserfläche beträgt $2 \%$. Insgesamt wurden für den Nationalpark 920 Pflanzenarten nachgewiesen, darunter 18 Orchideen. Im Nationalpark soll sich das größte europäische Vorkommen des Frauenschuhs (Cypripedium calceolus) befinden. Von den Gefäßpflanzen sind 67 geschützt und stehen 45 auf der Liste der gefährdeten Arten Polens. Ferner kommen geschützte Arten wie z. B. Schachbrettblume (Fritillaria meleagris), Moorveilchen (Viola persicifolia) oder Blattlose Schwertlilie (Iris aphylla) vor.

In den Sumpfwäldern kommen, je nach Feuchte und Mineralstoffgehalt des Untergrundes, vor allem Schwarzerle (Alnus glutinosa), Weiß-

Abb. 5 (oben): Birkenwald, im Unterwuchs mit Sumpfporst. Abb. 6 (unten): Polemonium caeruleum.
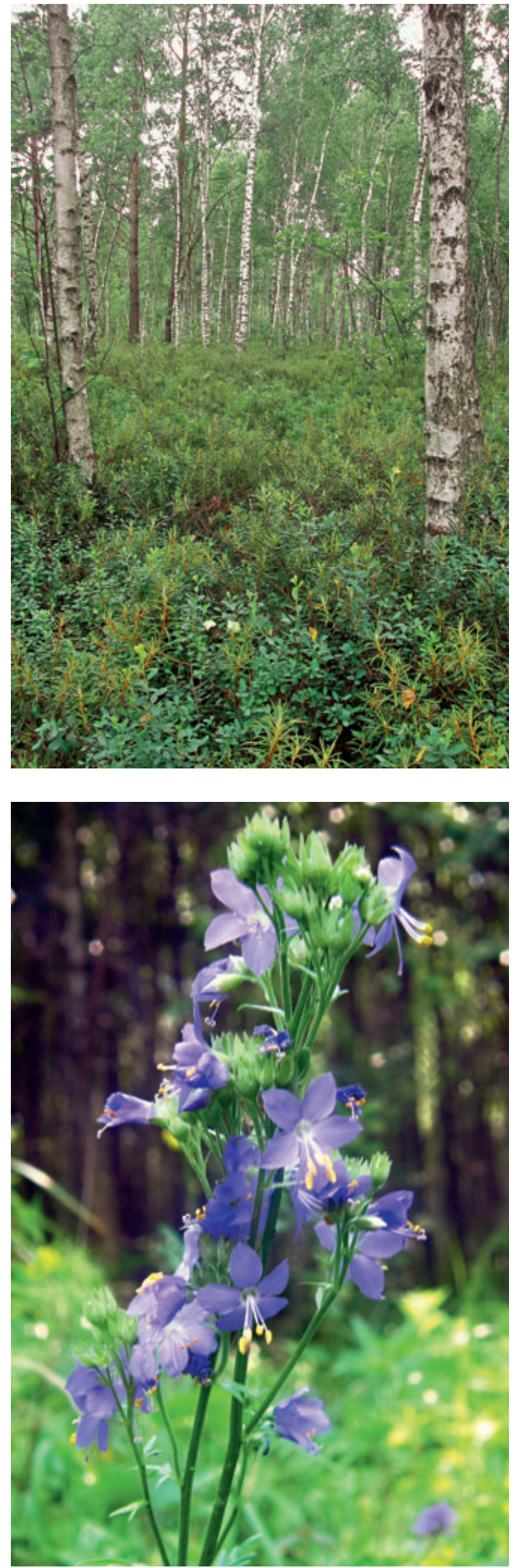

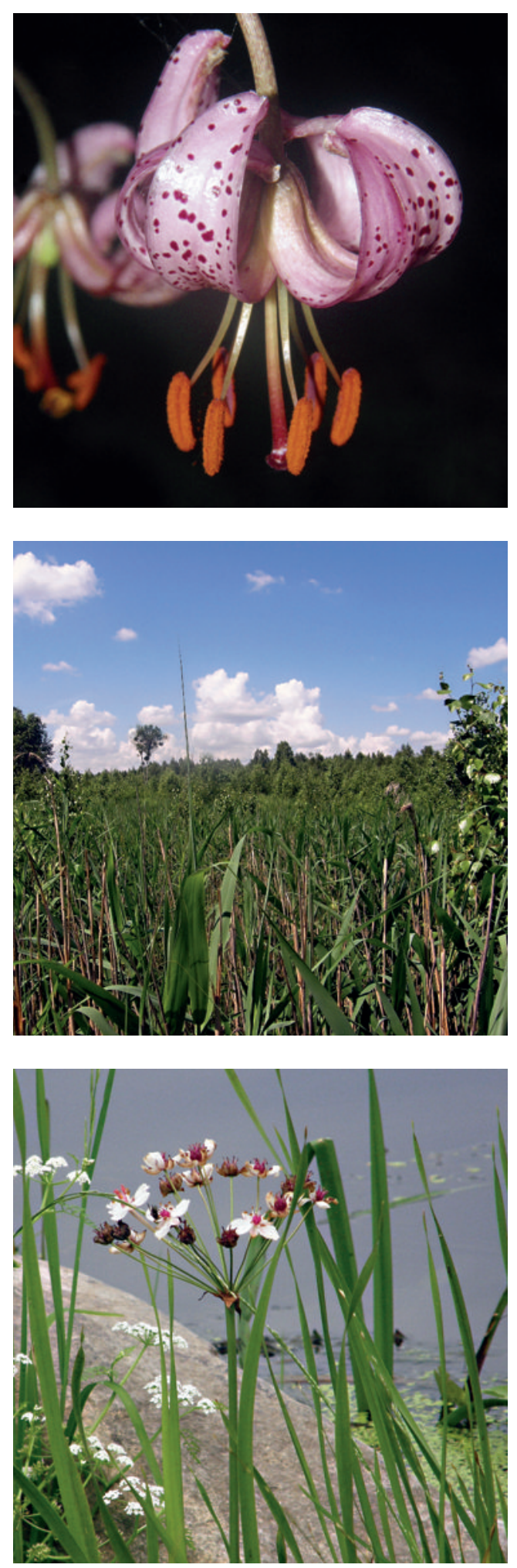

und Moorbirke (Betula pendula, B. pubescens), verschiedene Weiden (Salix), Zitterpappeln (Populus tremula) und Waldkiefern (Pinus sylvestris) vor. In der Krautschicht sind z. B. Sumpfdotterblume (Caltha palustris), Gelbe Schwertlilie (Iris pseudacorus) sowie verschiedene Farne, drunter auch der Sumpffarn (Thelypteris palustris) anzutreffen. An moorigen, torfmoosreichen und mineralstoffarmen Standorten wächst der Sumpfporst (Ledum palustre). Im Hochsommer ist von seinen zahlreichen weißen Blüten nichts mehr zu sehen. Dafür verströmen seine azaleenähnlichen Blätter beim Zerreiben einen würzigaromatischen Duft. Seine Blätter wurden früher zum Würzen von Bier verwendet. Als weitere typische Moorbewohner sind hier Vaccinium myrtillus, $V$. oxycoccus und $V$. vitis-idaea anzutreffen. Auf feuchten Flächen mit geringer Vegetationsbedeckung siedelt Sonnentau (Drosera rotundifolia). An den etwas trockeneren Waldund Wegrändern trifft man auf auch bei uns häufige Arten wie Acker-Witwenblume (Knautia arvensis), Wald-Erdbeere (Fragaria vesca) oder Bärenschote (Astragalus glycyphyllos).

Birken-Moorwälder, wie sie in Skandinavien häufig in der subborealen Zone vorkommen, sind in Polen eher die Ausnahme. Im BiebrzaNationalpark bilden sie meist lockere, lichte Bestände, in denen neben der Moorbirke auch die niedrige, nur bis $3 \mathrm{~m}$ hohe Strauchbirke (Betula humilis) vorkommt. Diese Birke ist ein Relikt aus der Eiszeit. Häufig bildet sie auch Gebüsche zusammen mit der Kriech-Weide (Salix repens) oder dringt in feuchte Wiesen ein. In der Strauchschicht der Sumpfwälder gedeihen als typische Gräser Pfeifengras (Molinia caerulea) und Sumpf-Reitgras (Calamagrostis canescens).

Besonders im Kernbereich des Nationalparks sind in den lichten Sumpfwäldern einige Raritäten zu entdecken. Zwischen verschiedenen Seggen-Arten fallen hier die uns aus den Alpen bekannte Türkenbund-Lilie (Lilium martagon) und die intensiv duftende Prachtnelke (Dianthus

Abb. 7 (oben): Lilium martagon.

Abb. 8 (Mitte): Sukzession zum Birkenwald.

Abb. 9 (unten): Butomus umbellatus. 


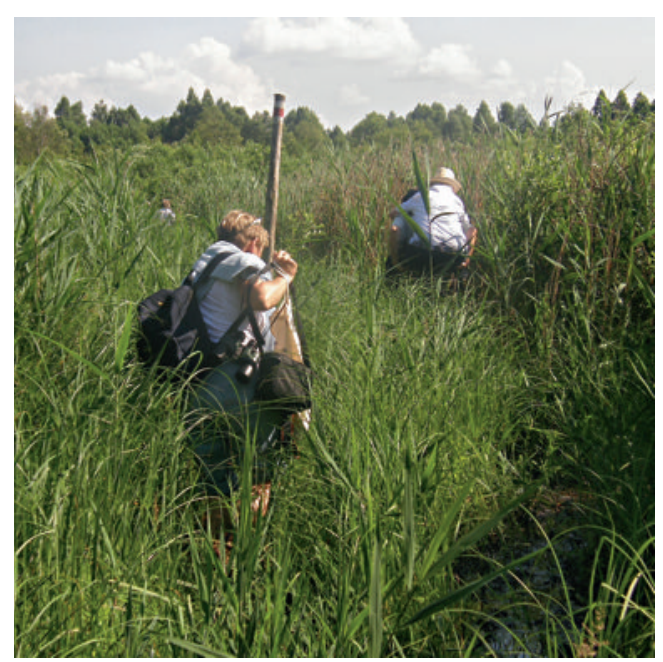

superbus) auf. Sowohl im Wald als auch auf Feuchtwiesen tritt die Himmels- oder Jakobsleiter (Polemonium caeruleum) als ein Relikt der Eiszeit auf. In Polen ist sie vom Aussterben bedroht.

Vom Rand des Waldes schweift der Blick weit über die ausgedehnten Sümpfe. An vielen Stellen ist eine Sukzession zum Birkenwald, gemischt mit Zitterpappeln, Weiden und später Kiefern, zu beobachten. Wesentliche Ursache ist die Aufgabe der extensiven Nutzung der Feuchtwiesen als Weideland oder Heuwiesen, wie es noch vor ca. 30 Jahren üblich war. Da die wenigen Bauern diese mühsame Bewirtschaftung nicht mehr leisten können, versuchen Naturschützer, zusätzlich durch Mähen zumindest einige der Feuchtwiesen baumfrei zu halten, denn gerade die offenen Lebensräume bieten vielen seltenen Tieren und Pflanzen einen letzten Rückzugsort.

Auf den nassen Wiesen oder entlang von Gräben fallen im Sommer vor allem Blut- und Gilbweiderich (Lythrum salicaria, Lysimachia vulgaris), Gelbe Wiesenraute (Thalictrum flavum), Schwanenblume (Butomus umbellatus) und das Fleischrote Knabenkraut (Dactylorbiza incarnata) auf. Im Biebrza-Nationalpark gibt es von dieser Orchidee die größte Population Polens. Im Frühling ist es ein überwältigender Anblick,

Abb. 10 (links): Abenteuerlicher Marsch durch einen feuchten Sumpf.

Abb. 11 (rechts): Lockere Vegetation auf den Sanddünen neben dem Sumpf.

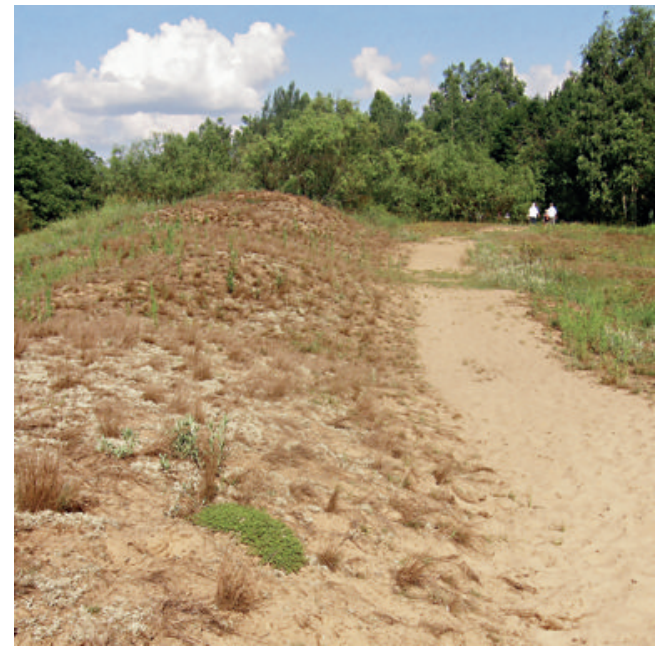

wenn die Sümpfe von einem gelben Blütenmeer der Sumpfdotterblume (Caltha palustris) überzogen sind. Eine Besonderheit der SumpfwiesenFlora ist der Moorkönig (Karlszepter, Pedicularis sceptrum-carolinum). Diese Läusekraut-Art hat hellgelbe, fleischige Blüten. Diese werden gern von Elchen abgefressen, so dass blühende Exemplare nur selten anzutreffen sind. Die stark bedrohte Art ist ein Relikt aus der Eiszeit.

Die Durchquerung von Randbereichen der feuchtesten Sümpfe kann recht abenteuerlich sein, da hier selbst im Sommer das Wasser durchaus über knöchelhoch stehen kann. Trotz der mitunter scharf schneidenden Blätter von Seggen und Wollgras empfiehlt es sich barfuß zu laufen, da sich Schuhe leicht im Schlamm festsaugen können. Seggen und Wollgras (Eriophorum angustifolium) bilden einen dichten Teppich. Dazwischen schieben sich die glatten Triebe des Teich-Schachtelhalms (Equisetum fluviatile) oder die Stängel von übermannshohen Schilfpflanzen (Phragmites australis). Auffällige Blütenpflanzen sind Sumpfblutauge (Comarum palustris), Zungen-Hahnenfuß (Ranunculus lingua) und Fieberklee (Menyanthes trifoliata), eine traditionelle, als Fiebersenkungsmittel genutzte Heilpflanze. Überraschenderweise kann der Wanderer an wenigen Stellen ein kontrastreiches Vegetationsmosaik erleben, wo direkt an den Sumpf bis $8 \mathrm{~m}$ hohe Binnendünen (z. B. Wilcza Góra) mit trocken- und wärmeliebender Vegetation anschließen. Die Dünen sind nur locker bewach- 


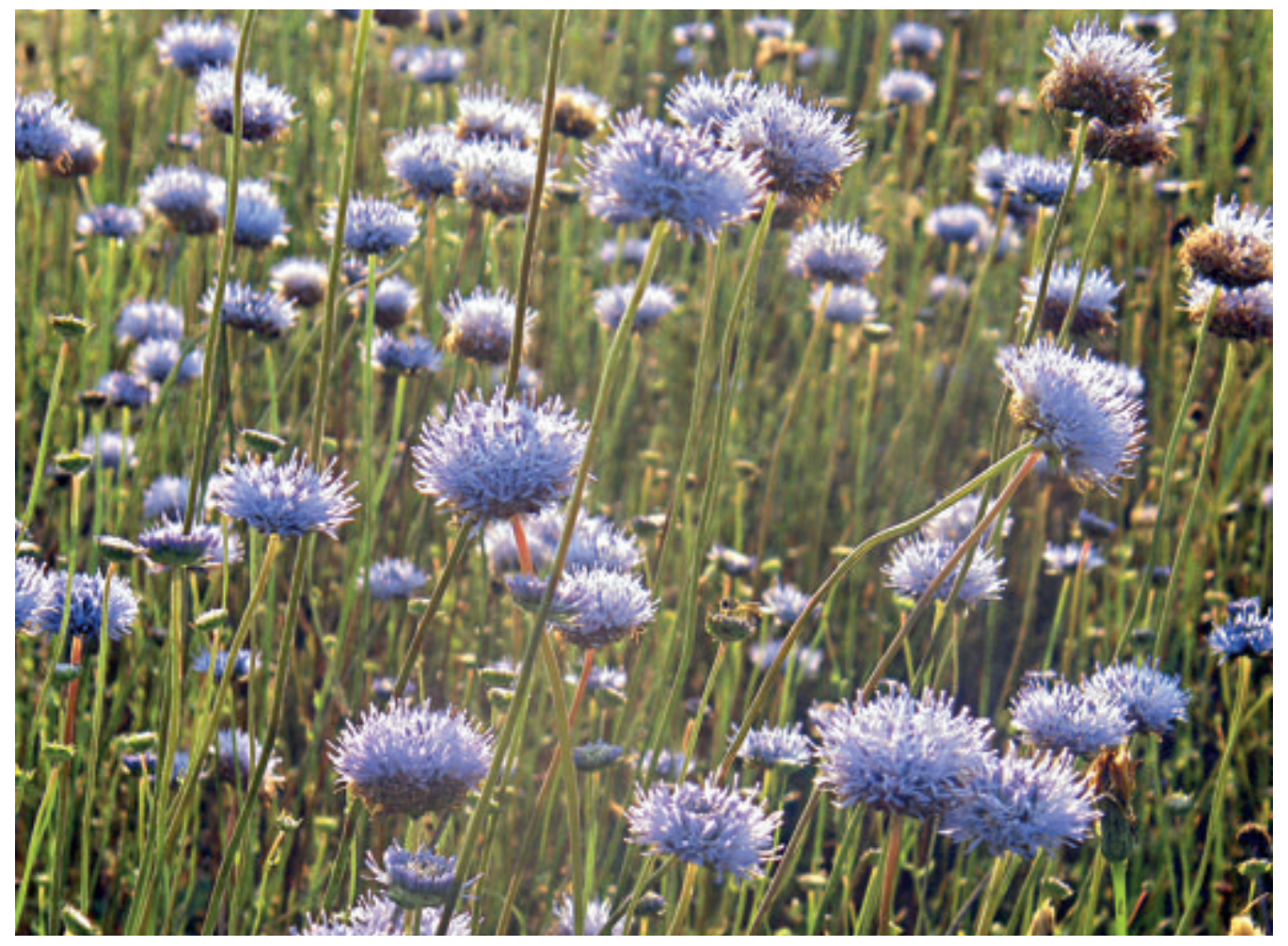

sen, so dass der freie Sand sichtbar wird. Wie für viele Dünengebiete typisch hat sich stellenweise eine Silbergrasflur mit Corynephorus canescens ausgebildet. Im Sommer fallen zudem große Bestände eines kräftigen Mauerpfeffers (Sedum maximum) auf, der leuchtend gelb blühende Polster bildet. Dazwischen findet man Strohblume (Helichrysum arenarium), Natternkopf (Echium vulgare), reichlich Acker-Rittersporn (Consolida regalis) und große Bestände des Sandglöckchens (Jasione montana). An lichten Stellen entwickeln sich Gruppen von Thymian (Thymus serpyllum), Mausohr-Habichtskraut (Hieracium pilosella), Heidekraut (Calluna vulgaris) und Borstgras (Nardus stricta).

Als Gehölze treten auf der Düne und vor allem an ihren Randbereichen Wacholder, Birken, Haseln und Kiefern auf. Unter den Kiefern findet sich Waldschilf (Calamagrostis epigejos), Adlerfarn (Pteridium aquilinum) und Preiselbeere (Vaccinium vitis-idaea). Am Waldrand ist auch die geschützte Arnika (Arnica montana) häufig.

\section{Tierwelt}

Bis zu 47 Säugetier-Arten leben im Nationalpark, die meisten davon sind Nager. Mittlerweile wieder weit verbreitet ist der Biber, die für den Fluss namensgebende Art. Mit einem Körpergewicht von $30 \mathrm{~kg}$ sind Biber die größten Nager des Gebietes. Sie waren früher in der Region sehr häufig, wurden aber von Wilderern ausgerottet. Erst im Jahr 1945 wurden wieder Biber aus Russland eingeführt, worauf sich die Population stark vergrößerte. Auch wenn der Beobachter nicht immer direkt ein Tier zu sehen bekommt, Bissspuren an Bäumen oder auch Biberburgen im Sumpf werden mit großer Wahrscheinlichkeit gesichtet.

Das markanteste Raubtier im Nationalpark ist der Wolf; 2-3 Rudel mit insgesamt über einem Dutzend Tieren sind dokumentiert. Die scheuen Tiere bekommt man nur selten zu sehen, ihre Spuren dagegen sind häufig. Die Wahrschein-

Abb. 12: Jasione montana. 
lichkeit ist groß, Wolfs-Spuren oder Kot in den Dünengebieten von Wilcza Góra zu finden, da hier regelmäßig einige Tiere herumstreifen. Das markanteste Großtier ist der Elch. Elche halten sich bevorzugt in den Sumpfgebieten auf, wo sie exzellent getarnt sind, aber von Beobachtungskanzeln aus gut beobachtet werden können. Auf das Vorkommen von Elchen bezieht sich auch der Name Elk für einen Nebenfluss des BiebrzaFlusses. Elche können über eine Minute unter Wasser bleiben, 5-6 m tief tauchen, um Wurzeln und Rhizome der Sumpfpflanzen zu beweiden. Im Winter bilden sie Gruppen, verlassen den Sumpf und halten sich bevorzugt in den Kiefernwäldern auf. Momentan leben etwa 500 Elche im Nationalpark, der größte Elch-Bestand Polens existiert im Roten Sumpf. Von diesem Areal breiteten sich die stark dezimierten Elche in ganz Polen wieder aus.

Ausgestorben, aber wieder rückgezüchtet wurde das Europäische Wildpferd (Tarpan). Die kleinen graubraunen Pferde mit dunkler Rückenzeichnung werden im Nationalpark weiter gezüchtet.

Im Biebrza-Flusstal leben 270 Vogelarten, von denen 180 dort auch brüten, darunter 2000 Paare des in Polen vom Aussterben bedrohten Seggenrohrsängers. Weitere markante Arten sind Doppelschnepfe, Blaukehlchen und Schwarzstorch. Allgegenwärtig sind die vielen Weißstörche, die wie im bekannten Kinderlied rotbeinig durch die Sümpfe waten. Im Sommer hat jedes Dorf bewohnte Storchennester aufzuweisen. Besonders in den Morgenstunden lassen sich auf den Sumpfwiesen auch Kraniche beobachten, deren etwas unheimlich klingende Rufe die Landschaft weit durchdringen.

Neben diesen spektakulären Tieren fasziniert ebenso die Welt der Kleintiere. Das Biebrza-Flusstal gehört zu den insektenreichsten Gebieten Polens. Hier kommt auch der seltene Schwarze Apollo (Parnassius mnemosyne) vor. Seine Raupen leben ausschließlich von Lerchensporn (Corydalis). Deutlich wird die Insektenvielfalt, wenn man

Abb. 13 (oben): Tarpan und Storch auf einer Feuchtwiese.

Abb. 14 (unten): Elchkuh mit Kalb. in der Frühe entlang der Sümpfe auf Beobachtungstour geht. Die meist noch klammen Insekten sitzen dann oft zu mehreren dicht gedrängt an diversen Blüten.

Im Land des Bernsteins ist auf Sumpfwiesen häufig die Bernsteinschnecke (Succinea putris) anzutreffen. Ihr Name bezieht sich auf die Farbe ihres Gehäuses. Bernsteinschnecken werden von einem Saugwurm (Leucochloridium paradoxum) parasitiert. Der über Vogelkot aufgenommene Parasit vermehrt sich ungeschlechtlich in der Schnecke und verursacht ein Anschwellen der Fühler. Vögel halten die dicken grün-weiß geringelten Fühler für Raupen und fressen die
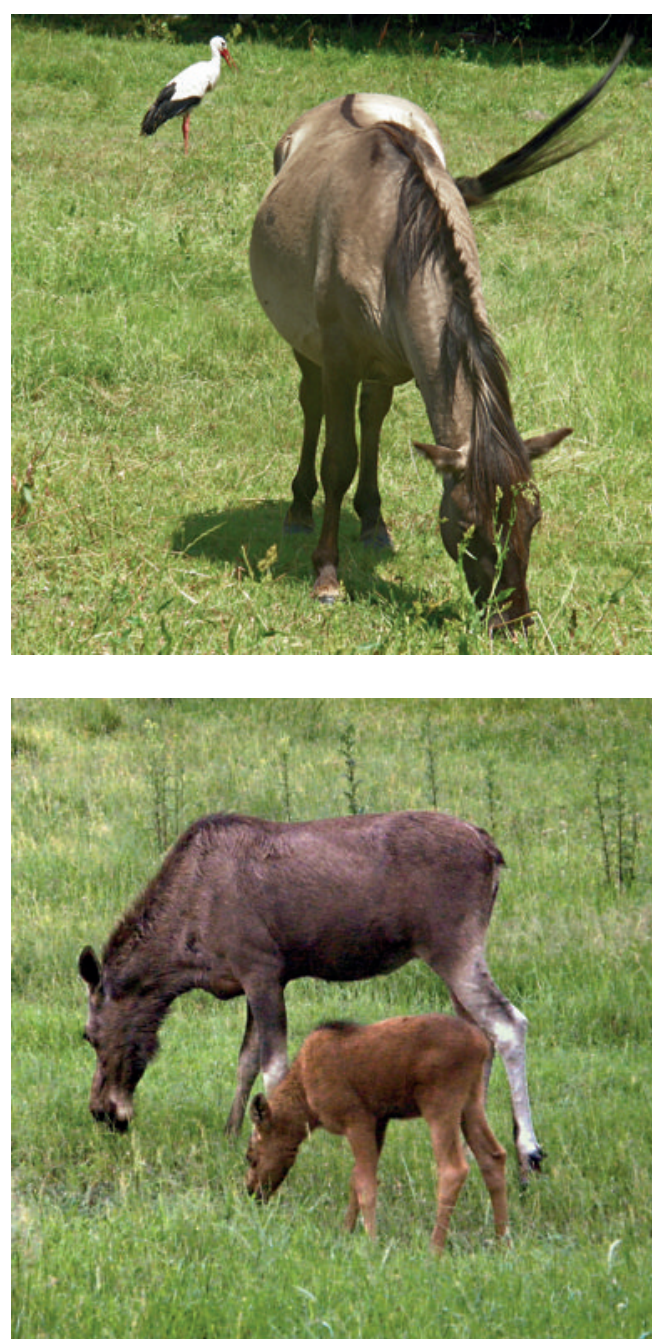

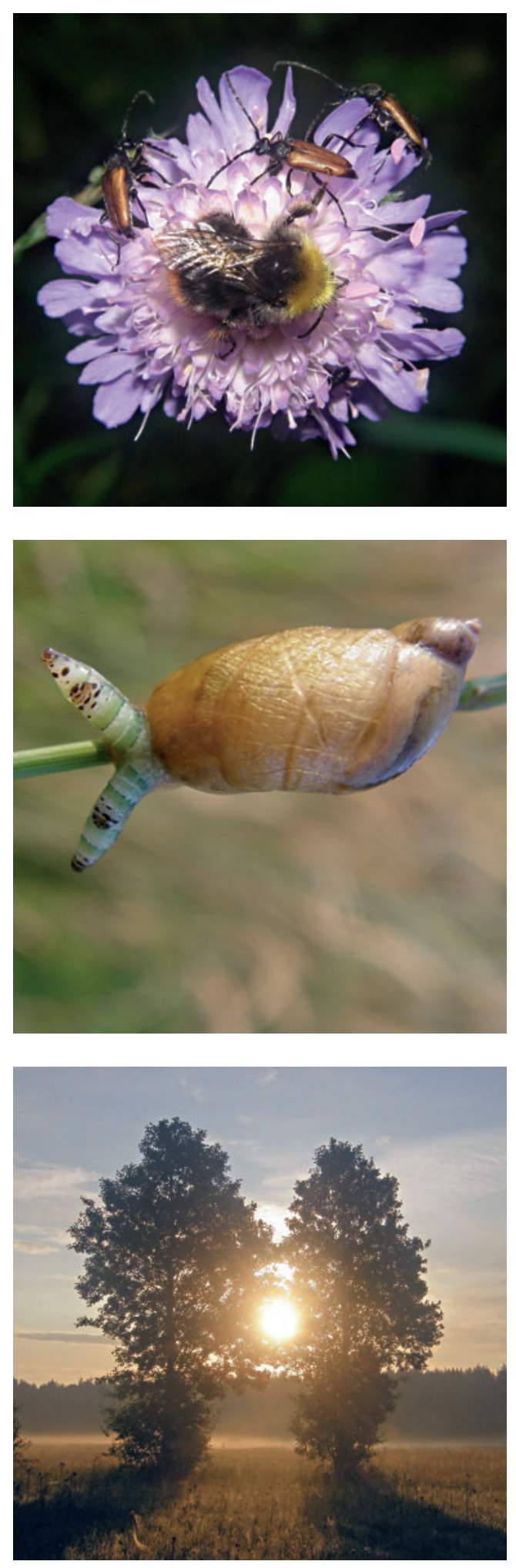

Schnecke oder nur die Fühler. Somit gelangt der Parasit in seinen Hauptwirt, den Vogel, wo er sich geschlechtlich vermehrt. Seine Eier werden mit dem Vogelkot ausgeschieden und gegebenenfalls wieder von einer Bernsteinschnecke aufgenommen, so dass sich der Kreislauf schließt.

Es bleibt zu hoffen, dass die Sumpfwiesen auch in Zukunft erhalten bleiben und weiterhin vielen seltenen Arten einen Lebensraum bieten. Für den geübten Naturbeobachter ist es äußerst spannend, nicht nur den spektakulären Tier- und Pflanzenarten aufzulauern, sondern auch im Unscheinbaren spannende Phänomene zu entdecken, was im Biebrza-Nationalpark sehr gut möglich ist. Man muss gar nicht um die halbe Welt reisen, um noch ursprüngliche Naturlandschaften kennen zu lernen, eine Exkursion in eines unserer Nachbarländer lässt staunen.

\section{Literatur}

WiATR, A. \& WRÓBLEWSKI, W. 2005:

Biebrza National Park. - Warschau.

\section{Internetseiten:}

www.wwf.de/fileadmin/fm-wwf/pdf-alt/regionen/ biebrza.pdf

(Hintergrundinformationen WWF-Projekt

Biebrza Nationalpark)

http://www.info-polen.com/natur/biebrza.php

Abb. 15 (oben): Überfüllung auf dem Blütenkopf einer Knautia arvensis.

Abb. 16 (Mitte): Parasitierte Bernsteinschnecke mit verdickten raupenähnlichen Fühlern.

Abb. 17 (unten): Morgenstimmung im Sumpf. 